\title{
Cosmic Evolution of Quasar Host Galaxies
}

\author{
D. Floyd ${ }^{1} \dagger ;$ M. Kukula, J. Dunlop, R. McLure ${ }^{2}$; C. O’Dea, \\ and S. Baum ${ }^{3}$ \\ ${ }^{1}$ STScI, USA ${ }^{2}$ ROE, UK ${ }^{3}$ RIT, USA
}

\begin{abstract}
Using HST WFPC2 and NICMOS observations, and our 2D image weighting and modelling technique (Floyd et al. 2004), we have reliably disentangled host from nucleus for nine optically matched radio-loud quasars (RLQ) and nine radio-quiet quasars (RQQ) at $z=1 \& 2$, in two bands spanning the $4000 \AA$ Abreak. The resulting galaxy colours provide the first unbiased estimates of galaxy mass for a statistical sample of quasars at high redshift, and indicates a difference in the evolution of radio-loud and radio-quiet objects.
\end{abstract}

Keywords. galaxies: active, galaxies: fundamental parameters, (galaxies:) quasars: general.

We revisit the sample of Kukula et al. (2001), consisting of 18 QSOs at $z \sim 1 \& 2$. Each epoch has equal numbers of RLQs and RQQs that are optically matched to ensure no a priori selection bias. We used NICMOS $J$ and $H$ band imaging in Kukula et al. (2001) to explore the rest-frame $V$ hosts. Here we add WFPC2 $R$ and $I$-band imaging (Floyd et al. in preparation) to explore the rest-frame $U$. We follow the observing and modelling strategy of Floyd et al. (2004) with deeper integrations of 3600-7800 s. Both the RL and RQ hosts follow roughly passive evolution of $1-4 L^{\star}$ populations (Fig. 1). However, within our sample, the RQQ hosts in general have bluer colour than their RL counterparts, indicating somewhat higher star-formation rates. This implies that the RLQ hosts have already formed the majority of their stars at an earlier epoch, while the RQQ hosts are still star-forming.RLQs are present in more massive, older systems and/or they can actively truncate star-formation in their hosts.

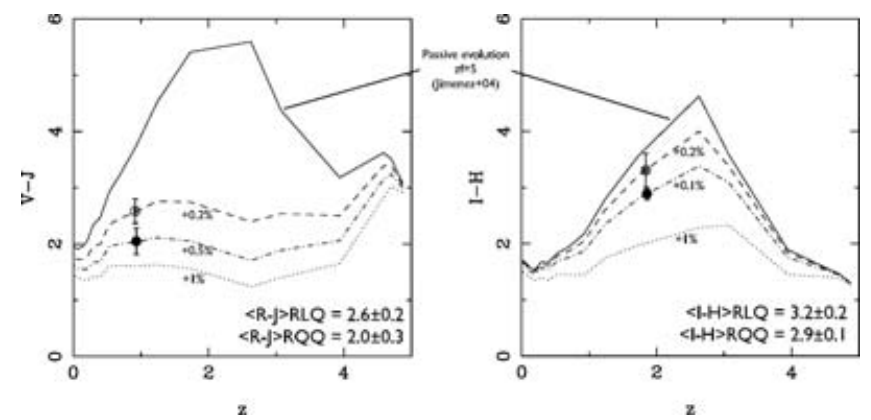

Figure 1. Mean R-J and I-H against $\mathrm{z}$ for RLQ (open) and RQQ hosts (filled). A difference in host colour is observed at each redshift. Passive evolution (solid line), plus Jimenez et al. (2004) SSP "frosting" models (\% mass in ongoing star-formation indicated) shown for comparison.

\section{References}

Dunlop, J. S., et al. 2003, MNRAS 340, 1095 Jimenez, R., et al. 2004, MNRAS 349, 240

Floyd, D. J. E., et al. 2004, MNRAS 355, 196 Kukula, M. J., et al. 2001, MNRAS 326, 1533

$\dagger$ email: floyd@stsci.edu 